\title{
A case of acute epiglottitis
}

\author{
B H M N Katugaha ${ }^{1}$, Srimathi Weerasekara ${ }^{2}$, Sujeewa Andrahennadi ${ }^{3}$, Subashini Basnayaka ${ }^{3}$
}

Sri Lanka Journal of Child Health, 2002; 31: 126-7

(Key words: acute epiglottitis)

\section{Introduction}

Epiglottitis is the term used for an inflammatory condition that involves not only the epiglottis but also adjacent tissues, including the ventricular bands, arytenoids and aryepiglottic folds ${ }^{1}$. It is almost always caused by the Haemophilus influenzae type $b^{2}$. Other major manifestations of H.influenzae type $b$ infection, such as meningitis or arthritis are rarely associated with epiglottitis ${ }^{3}$.

\section{Case report}

A four and a half year old boy was admitted to the paediatric unit of the Teaching Hospital, Kurunegala at about $5.30 \mathrm{pm}$ with difficulty in breathing following a respiratory infection. On examination, it was noticed that the child was terrified and prostrated, as he was struggling for his breath. Drooling and stridor were apparent and there was moderate fever and mild cyanosis. Acute epiglottitis was diagnosed immediately and the child was handled with utmost care to prevent a provocation of acute respiratory arrest. Oxygen was given via a facemask. Examination of the throat was never attempted. A lateral x-ray of the neck, taken with caution, showed a widened laryngeal air shadow with 'thumb print' appearance of the swollen epiglottis (Figure 1).

The Consultant Anaesthetist, Consultant ENT Surgeon and the operating theatre were informed of the case so that adequate preparation could be made for immediate tracheal intubation or, failing that, emergency tracheostomy. While waiting for the experts to arrive, the ward staff were ready with equipment for endotracheal intubation and, as expected, the child went into sudden respiratory arrest. The child was efficiently intubated by the experienced Relief House Officer, saving the life of the child. He was transferred to the Intensive Care Unit for ventilation, which was continued for four days. Antibiotics were given and the child made an uneventful recovery.

${ }^{1}$ Consultant Paediatrician, ${ }^{2}$ Relief House Officer,
${ }^{3}$ Senior House Officer, Teaching Hospital,
Kurunegala.

(Received on June 4, 2002)

\section{'Thumb Print' appearance of the swollen epiglottis.}
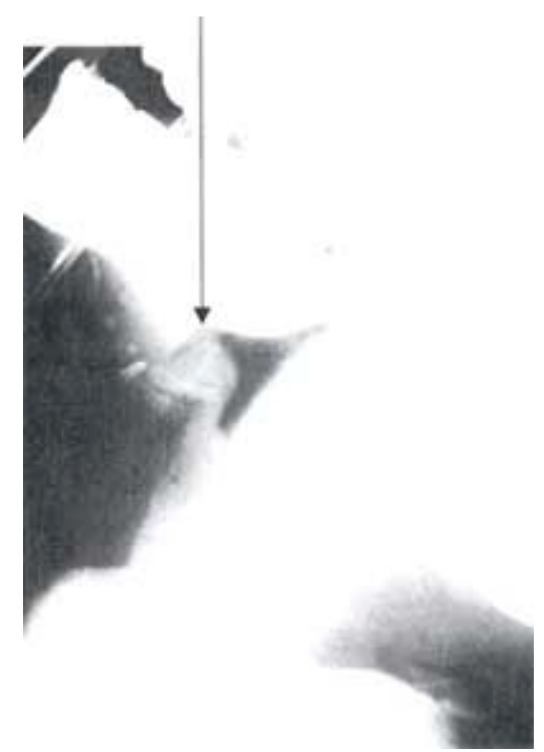

Figure 1 Lateral $x$-ray of neck showing widened laryngeal air shadow with 'thumb print' appearance of the swollen epiglottitis.

\section{Discussion}

Acute Epiglottitis is a potentially lethal, dramatic medical emergency in paediatric practice. Unless patients are handled with care, a fatal outcome may result. If suspected, direct visualization of the throat, with or without using a tongue depressor, should not be attempted unless an expert team is ready for immediate tracheal intubation or tracheostomy ${ }^{1}$. The choice of procedure is nasotracheal intubation, rather than tracheostomy, to avoid surgery and the somewhat longer period of recovery ${ }^{4}$.

This case report clearly highlights the fact that all doctors involved in paediatric practice should possess the skill of endotracheal intubation. Doctors, including intern medical officers, who opt to work in paediatric units, should be trained by experienced seniors in intubation before they are given "responsibility" to attend resuscitation of newborns and older children on their own. 


\section{Acknowledgements}

We wish to thank all those who helped to save the life of this child and Dr. A. Pallewatte, Consultant Radiologist and Dr. Anura Rajapakha of the Provincial Director's office, Kandy for their assistance.

\section{References}

1. Davis H W, Gartner J C, Galvis A G, Michaels R H, Mestad P H. Acute airway obstruction: Croup and Epiglottits. Paediatric Clinics of North America 1981; 28(4): 859-80.
2. Margolis C Z, Colletti R S, Grundy C. Haemophilus influenzae type $\mathrm{b}$ : The etiologic agent in epiglottitis. Journal of Pediatrics 1975; 87: 322-3.

3. Molteni $\mathrm{R}$ A. Epiglottitis: Incidence of extraepiglottic infection. Pediatrics 1976; 58: 526-31.

4. Lewis J K, Gartner C, Galvis A G. A protocol for the management of acute epiglottitis. Clinical Pediatrics 1978; 17: 494. 
